\title{
Thomas Manns Bericht über seine Lungenoperation
}

\author{
Thomas Mann's Account of his Lung Operation
}

\section{Bibliografie}

Dol http://dx.doi.org/

10.1055/s-0034-1378073

Pneumologie 2014; 68: 752-757

(c) Georg Thieme Verlag KG

Stuttgart · New York

ISSN 0934-8387

\section{Korrespondenzadresse}

Dr. med. Robert Kropp

Liegnitzer Straße 5

36100 Petersberg

dr.robert.kropp@gmx.de

\section{Vorwort \\ $\nabla$}

Der Schriftsteller Thomas Mann erkrankte 1946, während seiner Exiljahre in den USA und während der Arbeit an seinem Roman „Doktor Faustus", an einem Lungenkarzinom und musste sich einer Teilresektion der rechten Lunge unterziehen. Hierüber hat er mehrfach kurz in seinen Tagebüchern berichtet, ausführlicher in seinem Essay „Die Entstehung des Doktor Faustus. Roman eines Romans". Aus diesem Buch wird im Folgenden das entsprechende Kapitel wiedergegeben. ${ }^{1}$ Welche Beweggründe haben uns zu diesem Entschluss geführt? Es ist sicherlich reizvoll, einen Blick auf die Thoraxchirurgie in der Mitte der neunzehnhundertvierziger Jahre zu werfen, auf die Usancen in der Klinik, auf das Gehabe der ärztlichen Kollegen jener Zeit (welches sich offensichtlich nicht sonderlich von heute unterscheidet), auf die Medikation, die Operation und perioperative Behandlung und Pflege und anderes mehr, und das aus der Sicht eines prominenten Laien, Patienten. Darüber hinaus kann der Leser mittels dieses Pneumologen-affinen Textes die wunderbare Sprache Thomas Manns, ihren Humor, ihre Anschaulichkeit und Exaktheit, auch ihre ironische Seite genießen.

Dem S. Fischer Verlag sei herzlich für die Nachdruckgenehmigung gedankt.

Hierbei wünschen wir Ihnen viel Vergnügen!

Professor Dr. Tom Schaberg

Dr. Robert Kropp

\footnotetext{
${ }^{1}$ Der ursprüngliche Gebrauch des „ß“ wurde beibehalten.
}

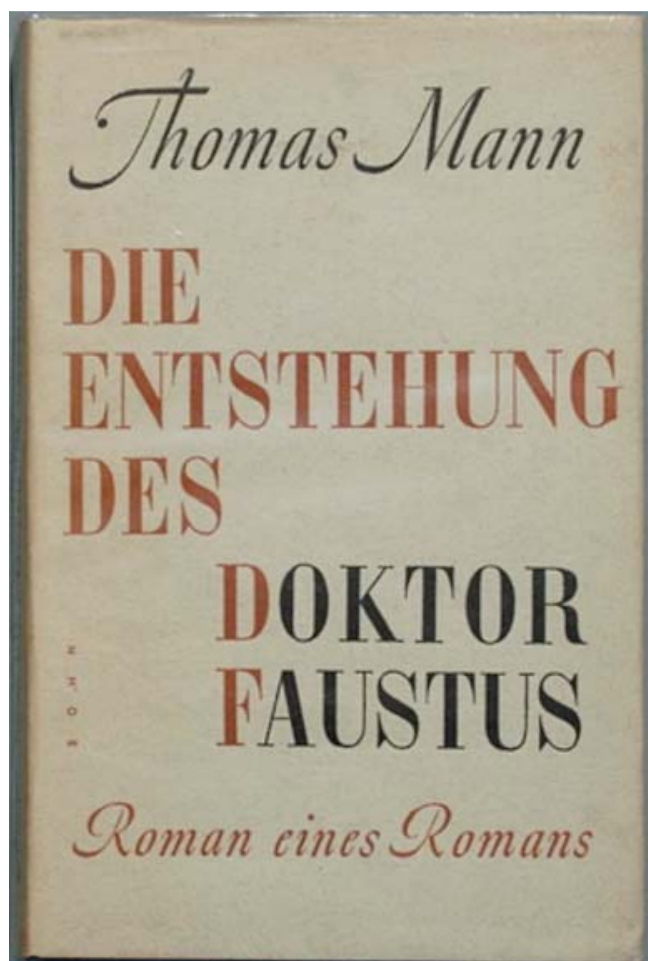

\section{Mann Th. Die Entstehung des Doktor Faustus.}

$\nabla$

Ende des Kapitels XII

Gegen Mitte Januar 1946 [...] schwankten meine Kräfte mehr und mehr, und im Tagebuch mehrten sich die trockenen Vermerke über Kopfschmerzen, Hustennächte, Nervenschwäche und „absurde“ Müdigkeit. [...] Röntgen-Aufnahmen meiner Lunge hatten einen „Schatten“ irgendwo in diesem Organ zum Vorschein gebracht, von dem der Doktor meinte, daß man gut täte, ihn weiter zu beobachten. Vorderhand empfahl er die Behandlung von Nase und Rachen durch einen Spezialisten, [...], der sein Bestes tat, Symptome zu mildern, deren sekundärer Charakter immer deutli- 


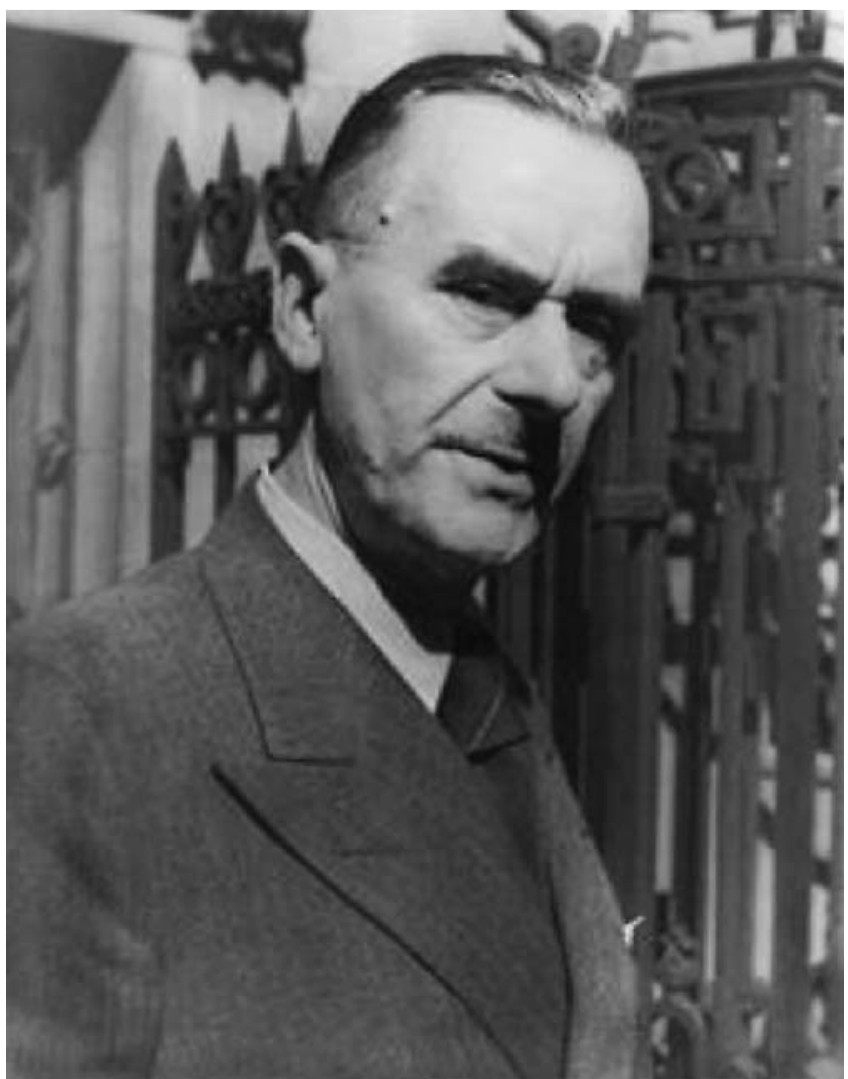

Thomas Mann.

cher wurde. Längst hatte ich, nur halb eingestandenermaßen, nachmittags und abends ein bißchen erhöhte Temperatur, hatte auch welche, als ich [...] zusammen mit meinem Bruder $^{2}$ einen Rezitationsabend besuchte, den Ernst Deutsch im Warner-Studio veranstaltete. [...] Ich genoß den Abend sehr, in dem etwas verfremdeten, zugleich matten und gehobenen Zustand, in den ein mäßiges Fieber versetzt. Spät kam ich zu Bette - und verließ es einige Tage nicht, da eine grippige Erkrankung, nachmittags immer $39^{\circ}$ Fieber erzeugend, mich darin festhielt. Eine Tag und Nacht alle drei Stunden durchgeführte orale Penicillin-Kur schlug überhaupt nicht an. Besser half immer die Empirin-KodeinMischung. Ich schlief viel, auch am Tage [...].

Ein Hin und Her von halber Genesung und Rückfällen in fiebrige Zustände folgte. Ich fuhr und ging etwas aus, aber es wollte nicht gut tun, und namentlich die sonst so geliebte Seebrise schadete mir. Zum Tee sah ich Gäste, aber meine Frau brachte dann wohl mit Kopfschütteln das oben liegengelassene Thermometer, das wieder mehr als 38 zeigte, und schickte mich zu Bette. [...] Damals übernahm Dr. Friedrich Rosenthal meine Behandlung. Er wandte Injektionen meines eigenen Blutes an, die nichts fruchteten, und versuchte es dann mit einer Empirin-Bellergal-Kur, um kein Fieber mehr aufkommen zu lassen. Unterdessen hatte er die letzten Röntgen-Aufnahmen der Lunge eingefordert, die ihm das klare Bild einer Infiltration am rechten Unterlappen ergaben. Er verlangte die Zuziehung eines Spezialisten, der, Amerikaner, den Befund durch Untersuchung bestätigte und die bronchoskopische Feststellung des Abszesses beantragte, auch schon die Notwendigkeit einer Operation durchblicken ließ. Ich war mehr ver-

${ }^{2}$ Heinrich Mann, älterer Bruder Thomas Manns. wundert als erschrocken, denn nie hatte ich gedacht, daß mir von den Atmungsorganen her irgendwelche Gefahr drohen werde, und die einhellige Versicherung der Ärzte ging denn auch dahin, daß es sich um keinen tuberkulösen Prozeß handelte.

\section{Kapitel XIII}

Der energische Fortgang der Dinge, wie sie sich nun, und zwar glücklich, entwickelten, ist allein meiner Frau zu danken, die von uns allen als einzige wußte, was sie wollte, und das Notwendige einleitete. Rosenthal war im Grunde, meines Alters wegen, gegen die Operation, ja auch, um der Schonung willen, gegen die Bronchoskopie, von der der amerikanische Doktor gleichmütig gemeint hatte, in acht Tagen werde ich mich davon erholt haben. Sein behandelnder Kollege war aus lauter Menschlichkeit nicht ungeneigt, es auf die Resorption des Abszesses durch einen im Ganzen ja gutwilligen Organismus, ohne Eingriff, ankommen zu lassen, und für diesen Weg schien der Erfolg einer PenicillinInjektionskur zur sprechen, die von einer nun eingestellten Pflegerin achtmal in vierundzwanzig Stunden durchgeführt wurde. Die Droge beseitigte das Fieber vollkommen, und ich habe während der ganzen Affäre nie wieder welches gehabt. Dennoch wußten wir alle, daß mit der Methode des Zuwartens das nicht geringe Risiko größter Ungelegenheiten verbunden blieb, und während der Doktor zögerte und ich selbst es am bequemsten fand, über mich bestimmen zu lassen, hatte meine Frau ihre Entschlüsse gefaßt. Sie hatte sich mit unserer Tochter Borgese in Chicago, diese sich mit der Universitätsklinik, „Billings Hospital“, in Verbindung gesetzt, an der einer der ersten Chirurgen Amerikas, Dr. Adams, wirkt, als Pneumotom von besonderem Ansehen. Rasch war dort alles geordnet, hier für Eisenbahn-Reservation und Ambulanz-Transport zum Bahnhof gesorgt, und ehe ich mich's versah, fand ich mich vorm Hause, unter den bekümmerten Blicken unseres japanischen „couples“, Vattaru und Koto, auf der Bahre ausgestreckt, die in den weich und schnell gehenden Krankenwagen geschoben wurde.

Unter so neuartigen Umständen ging es nach Union-Station und, mit dem Vorrecht direkten Zuganges zum Zuge, vor unseren „bed-room“, auf dessen Lager ich im Schlafrock, mit meiner Frau als recht unbequem untergebrachter Beisitzerin, die nächsten sechsunddreißig Stunden verbrachte. Elisabeth erwartete uns in Chicago, und wieder stand dort eine Ambulanz bereit, deren Rollbahre mich bald, von Lifts gehoben, durch die weitläufigen Korridore von „Billings Hospital“ und in das bereitgestellte, von dem guten Kind schon mit Blumen geschmückte Zimmer führte. Wie lebhaft steht mir, der ich nie die Lebensordnung eines großen Krankenhauses erfahren, mit Chirurgie nie zu tun gehabt hatte, diese ganze Ankunft und Installierung vor Augen: die Bekanntschaft mit den durch die nicht bis zum Boden reichende Pendeltür hin und her schwebenden, messenden, injizierenden und alle Augenblicke kalmierende Tabletten verabfolgenden Pflegerinnen; die fast sofort erfolgende Begrüßungsvisite der behandelnden Ärzte in corpore, an der Spitze der Operateur Dr. Adams selbst, ein Mann von schlichter Liebenswürdigkeit und Herzensgüte, ohne eine Spur der Tyrannenallüren des Anstaltsgewaltigen deutschen Stils, vor dem Assistenten und Schwestern zittern; sodann sein „Medical Advisor“, der Internist und Universitätsordinarius, Professor Bloch, hochgewachsen, brünett, aus Fürth bei Nürnberg gebürtig, wie er mir bald auf deutsch vertraute; ein Dr. Philipps dazu, Lungenspezialist, scherz- und plauderhaft von Natur, ein erst vierundzwanzigjähriger Dr. Carlson, nordischer Herkunft und bildhübsch, „Intern“ dieses großen Hauses schon seiner ausgezeichneten Intelligenz und manuellen Geschicklich- 
keit wegen, - und weitere weißbekittelte Entourage. Es waren angenehme persönliche Eindrücke für den Anfang. Die erste Allgemeinuntersuchung nachher nahm Professor Bloch, mit Autorität eintretend, dem Assistenten aus der Hand, der schon damit begonnen hatte. Sein Verhör über die Vorgeschichte der Krankheit war freundschaftlich und genau. Sie wurde übrigens in stundenlanger Arbeit, nach dem Diktat meiner Frau, von jüngeren Herren zu Protokoll gebracht. Formell war der Entschluß zum operativen Eingriff noch nicht gefaßt, sondern abhängig vom Ergebnis der Bronchoskopie, das aber so ziemlich feststand.

Die Prozedur bildete eines der markanten Vorkommnisse der nächsten zehn Tage, die mich mit meinem ingeniös konstruierten, am Kopf- und Fußende in beliebige Höhe zu kurbelnden Hospitalbett und überhaupt mit der Lebensform des Patienten vertraut machten, mit dem Anstaltstage, der früh begann und früh endete. Auf einem „stretcher“ ausgestreckt und in den Elevator geschoben, gelangte ich in untere Räumlichkeiten, wo eine größere Anzahl entweder direkt an dem Erkundungsakt beteiligter oder des Zuschauens wegen gekommener Hausverwandten, darunter Freund Bloch, versammelt war. Die Schonung, mit der man vorging, war überraschend, höchst dankenswert und in ihren Mitteln zauberhaft. Eine anästhetisierende Pinselung des Rachens machte den Anfang. Dann, während mein Kopf im Schoß eines Assistenten lag (der ihn später wohl rasch emporzuheben hatte), bekam ich von einer weißbeschürzten Frauensperson energisch-tätigen Typs eine Injektion in die linke Armbeuge, nebst dem Bedeuten, daß ich nun sehr bald schläfrig werden würde. Schläfrig? Ich hatte nach dem Empfange kaum noch zwei Worte gesprochen, als mein Bewußtsein so sanft wie restlos entschwand und ich - wohl nur für kurze Frist, fünf oder sechs Minuten - tief ungewahr jedes Dinges war, das mit mir geschah. Was geschah, muß wachen Geistes recht peinlich hinzunehmen seinder kalifornische Consiliarius hatte ja gesagt, daß ich mich binnen einer Woche ganz gut davon erholt haben würde. Hier war Erholung nicht not, denn es gab keine Strapaze. Ich erwachte, schon wieder in meinem Zimmer, davon, daß der gute Dr. Adams, der mich hinaufbegleitet, mir mildtätig die Nase schnäuzte. Denn die Einführung des mit einem elektrischen Lämpchen versehenen Apparats durch die Luftröhre in die Lunge (wobei eine Art von periskopischer Vorrichtung erlaubt, sich genau über die Verhältnisse dort unten aufzuklären) bewirkt natürlich eine schleimige, leicht blutige, Reizung des ganzen Atmungstraktes, und man braucht nach der Rückkehr in sein Bett einige Papierservietten, was aber auch von Unannehmlichkeit alles ist. Ich war entzückt und sprach tagelang zur Erheiterung namentlich der jungen Mediziner, mit Bewunderung, Preis und Dank von der magischen Spritze.

Das noch nicht lange gebräuchliche Mittel heißt, wenn ich nicht irre, Pentathol, aber nie erfuhr ich an Ort und Stelle den Namen. Es gehört zu den wunderlichen Gesetzen und Schweigegeboten dieser Stätten, daß man nie erfährt, worin die Anwendungen bestehen, die einem geboten werden, und sehr bald lernt man, neugierige Erkundigungen danach als taktlos zu empfinden. Die Schwestern verweigern jede Auskunft über das Ergebnis der Temperaturmessungen. Nie würden sie verraten, woraus die weißen Plätzchen bestehen, die sie alle paar Stunden mit einem Glase Wasser servieren, noch würde ein Arzt Namen und Natur eines verordneten Medikamentes verraten. Ich erinnerte mich, wie ich mir während der Rekonvaleszenz mit gebackenem Fisch ein wenig den Magen verdorben hatte und noch spät abends den gerade diensthabenden „Intern“ hereinbitten ließ, um ihm meine Beschwerden zu klagen. Am besten, sagte ich, helfe mir in sol- chem Fall immer ein halber Teelöffel Natron bicarbonicum. Er überhörte das. Er erkundigte sich noch längere Zeit nach den Verstimmungssymptomen und ihrer möglichen Ursache. Schließlich sagte er: „Well, don't worry, we will give you a little something which will be helpful.” Die Schwester brachte das „little something" in einer Tasse. Es war Natron.

Die Operation war nun endgültig beschlossene Sache, und die nächsten fünf, sechs Tage galten, in Abwesenheit des Dr. Adams, der zu irgendeinem Ärztetreffen gereist war, den erdenklichen Vorbereitungen und Sicherstellungen. Blutentnahmen, Ausflüge im Rollstuhl oder auf dem „stretcher“ ins X-ray-Laboratorium, Besuche der verschiedenen Spezialisten des Hauses folgten einander. Besonders der für das Herz, ein Engländer, wenn ich mich recht erinnere, war hochbefriedigt. Mit dem Herzen, erklärte er, sei ich jeder Operation gewachsen. Eine sehr wichtige Persönlichkeit sprach vor: Dr. Livingstone, Gattin meines Operateurs und Vorsteherin aller Anästhesie, zauberische Mischerin tiefschlafbringender Fluiden. Sie mußte mir versprechen, die bewunderte Armspritze bei der Hauptoperation wieder ihren Segen wirken zu lassen. - Die Herstellung der Pneumothorax, will sagen des Einlassens von Stickstoff in die Brusthöhle zur Stilllegung des erkrankten Lungenflügels, kam auch an die Reihe, und es war doch merkwürdig, eine Applikation, die ich in vergangenen Arbeitstagen, zur Zeit des Zauberbergs, soviel im Munde geführt, am eigenen Leib zu erfahren. Professor Bloch nahm sie mit größter Akkuratesse und Geschicklichkeit vor, und der kleine Carlson sah lernbegierig zu. Das Ganze war kaum eine Unannehmlichkeit zu nennen, aber Bloch lobte mich sehr für mein kooperatives Verhalten, und als ich mich darüber wunderte, sagte er: „Wenn Sie wüßten, wie die Leute sich oft dabei anstellen!“ Unterdessen war Erika, auf die Nachricht von dem, was vorging, aus Nürnberg durch die Lüfte zu uns gestoßen, an die Seite ihrer Mutter, die bei Borgeses wohnte und die Mehrzahl der Tagesstunden an meiner Bettseite verbrachte. Nichts konnte uns beiden tröstlich willkommener sein, als die Anwesenheit des lebens- und liebevollen, stets heiteren Auftrieb bringenden Kindes. Sie ließ es sich angelegen sein, die Blumen zu warten und auszutauschen, mit denen mein Zimmer sich gefüllt hatte, - Vorschußlorbeeren, gespendet vor der Schlacht, aber eine Augenweide, auf die ich so stolz und erpicht war wie jeder Kurbelbetthabitant, - ,just another patient“, wie eine der Pflegeschwestern mich neugierig nachfragenden Bekannten beschrieben hatte. Fieberfrei und ohne Schmerzen, war ich eben nur sehr schwach, so daß schon Rasieren eine übergroße Anstrengung bedeutete und also die Bluttransfusion, die ich einen Tag oder zwei vor der Operation empfing, doch wohl nicht ganz überflüssig war. Zwei junge Praktikanten verabfolgten sie nach der Routine, und während die Blutkonserve langsam in meine Gefäße tropfte, unterhielt ich die Herren mit der Lektüre einer der erstaunlichen Neuigkeitsfabrikate, die Erika aus aufgeklebten Zeitungslettern und -worten herzustellen pflegte, eines „4-Power Showdown Triumph Bulletin 1946, released after Wild Ride for Germany“ mit solchen erregenden „headlines“ wie: „Truman sniffs at U.S.Policy“, „Eisenhower May Be Arrested on Spy Charge“, „Germany Demands Dismissal of U.S.Government. Explains Why", "Russia Asked to Neglect Red Defense", "Truman Hopes to Lure Stalin to Missouri, Pepper Says", “Foreign Born Babies by War, Navy Leaders Pose Problem - Ike Will Recognize Quintuplets - Bradley Favors Murder" etc. etc.... Es herrschte also eine nicht ganz schickliche Heiterkeit während des Aktes, aber mir lag dran, die jungen Leute lachen zu sehen, um selbst besser über das leicht Grausige der Sache hinwegzukommen. 
Dann war Adams zurück und erklärte, wenn ich nichts dagegen hätte, könnten wir „go ahead“. Morgen früh also. Meine Frau ließ es sich, ein wenig gegen die Hausordnung, nicht nehmen, die Nacht in dem recht unbequemen Lehnstuhl neben meinem Bett zu verbringen, während ich den Schlaf vollkommener Gemütsruhe schlief. Dennoch hatte ich noch nachmittags Dr. Bloch gefragt, wie „Lampenfieber“ auf englisch heiße. „Stage fright“, hatte er geantwortet. Punkt 7 Uhr, wie immer, wurde Tag und Toilette gemacht. Ich empfing meine „Hypo“ (vertrauliche Abkürzung für hypodermic injection; es war Morphium, natürlich, aber wer hätte zu fragen gewagt), und dann winkte ich von dem „stretcher“, der mich entführte, der zurückbleibenden Getreuen den Abschiedsgruß. Nie vergesse ich die sanfte Stimmung in dem halb dunklen Vorraum des Operationssaals, wo ich auf meiner Bahre eine Weile zu warten hatte. Es bewegten sich Leute um mich her, aber sie gingen auf Zehenspitzen, und wer zu kurzer Begrüßung an mich herantrat, tat es mit äußerster Zartheit. Bloch steckte den Kopf durch die Tür und nickte mir zu: „No stage fright today“, ließ ich ihn wissen, aber er ging auf meinen Humor nicht ein. Professor Adams wünschte Guten Morgen und kündigte mir an, daß ich außer der geliebten Armspritze auch noch etwas einzuatmen bekommen würde, „a little something“. Ich war gerührt von seiner Gewissenhaftigkeit. „Wohl kenn’ ich Irlands Königin“, zitierte ich bei mir und meinte die Livingstone. Die ließ sich denn auch bald bei mir nieder, machte sich zunächst an meinem Arm zu schaffen (vielleicht markierte sie nur; denn was liegt an einem bißchen Pentathol, wenn lange Arbeit bevorsteht) und setzte mir dann mit leichter Hand die mit edlen Stoffen getränkte Maske auf. Hinweg. Es war die friedlich-unbeängstigende und geschwindeste Narkose, die sich denken läßt. Ich glaube, ein einziger Atemzug genügte, mich in die gründlichste Abwesenheit zu versetzen, - der freilich gewiß während der nächsten anderthalb oder zwei Stunden mit öfterem Geträufel nachzuhelfen war. Ich hatte, soviel ich weiß, nicht teil daran, aber nach allem, was ich später darüber hörte, waren es begünstigte Stunden. Es war ein schöner Morgen, alle hatten so trefflich geschlafen, alle waren in frischer Lust und Laune tätig, voran Dr. Adams, der mit gewohnter, im Tempo genau gemessener, nie sich übereilender, aber durch die Exaktheit des Einzelgriffes dennoch zeitsparender Meisterschaft arbeitete. $\mathrm{Zu}$ Hilfe kam ihm eine geduldige Natur mit immer noch solidem Hintergrund (ich brauchte nur eine Bluttransfusion noch während der Handlung, da andere, und jüngere, zwei oder drei benötigen) und vereinigte sich mit entwickeltstem ärztlichen Können zu einem fast sensationellen klinischen Erfolg. Tagelang nachher soll in medizinischen Kreisen New Yorks und Chicagos von der „most elegant operation“ die Rede gewesen sein.

Meine Frau, Erika und Medi verbrachten die Stunden vertrauensvoller Spannung in Dr. Blochs „office“. Von Zeit zu Zeit kam er, ihnen Bericht zu erstatten. „Es geht gut, es geht sehr gut“, sagte er, und seine Hand war kalt. Dann hatte meine Frau mich im Zimmer erwartet, wo ich, längst wieder in meinem Bett, vorübergehend erwachte. Noch stark benommen sprach ich gegen alle Gewohnheit englisch zu ihr, und sonderbar! ich führte Klage. „It was much worse than I thought”, sagte ich. „I suffered too much!” Noch heute denke ich nach über den Sinn dieses Unsinns. Wovon redete ich? Ich hatte von allem ja nichts gespürt. Gibt es irgendwelche Tiefen des Vitalen, in denen man, bei völlig ausgeschaltetem Sensorium, dennoch leidet? Ist Leiden vom Erleiden im Untersten nicht vollkommen zu trennen? Dies könnte sich sogar auf den „toten“ Organismus beziehen, von dem niemand weiß, wie tot er vor seiner wirklichen Auflösung ist; es könnte, wenn auch als mißtrauische Frage nur, ein Argument gegen die Feuerbestattung bilden. Um englisch zu sprechen: „It may hurt“. -

Die Nachwirkungen der Narkose waren geringfügig, sie störten mich kaum im Weiter- und Weiterschlafen. Etwas warmes Wasser, auch kaltes zur Abwechslung, bekam ich durch eine Glasröhre zu trinken. Der Flüssigkeitsverlust bei solchem Eingriff ist bedeutend. Um sieben Uhr fragte ich den kontrollierenden Arzt nach der Zeit. Er nannte sie mir. „Sie sind früh auf“, wunderte ich mich. „Nicht ganz so“, erwiderte er. „Es ist noch derselbe Tag.“ Ich schlief schon wieder. Ich glaube, es war noch in dieser Nacht, oder morgens früh, daß ich Orangensaft durch die Glasröhre bekam. Nie im Leben hat mir etwas so köstlich geschmeckt. Es war ein wirkliches Entzücken. Offenbar wurden Durst und Hunger durch diese Darreichung gleichermaßen befriedigt, und es ist unglaublich, wie das unbewußte Bedürfnis des Körpers die Empfänglichkeit der Geschmacksnerven bis zur Wonne verstärkt. Mit ähnlicher Genußfähigkeit sollen diese auf irgendwelche Süßigkeit, ein gewöhnliches Praliné, nach der Anwendung von Insulin reagieren. - Ich hatte nun Privatpflegerinnen, ihrer drei, die in einem Tag- und Nacht-Turnus von je acht Stunden einander ablösten. Ihre Hauptobliegenheit war, außer den dreistündigen, Infektion verhindernden Penicillin-Gaben, der Beistand bei dem sehr mühsamen Umwenden im Bett, das immer wieder gefordert ist; denn Bewegung, Wechsel der Lage, bald so, bald so, ohne Bevorzugung der unverwundeten Seite, das gehört heutzutag zur Behandlungstechnik, und schon am zweiten Tag nötigte der junge Carlson mich, allerdings unter seiner zum Auffangen bereiten Hut, ein paar Minuten neben meinem Lager frei auf den Füßen zu stehen. Das ging ganz gut, und nur mit der Rückkehr über den Schemel in das ziemlich hohe Bett hatte es seine Schwierigkeiten. Die Schwester der Nachtstunden, von 11 bis 7, hieß June Colman, eine denkwürdig angenehme Person. Es ist fast unvermeidlich, daß die Gefühle des Patienten, sei er auch alt, vernäht und schwer umdrehbar, für den Engel seiner Nächte, ist dieser nur leidlich lieblich - und June war entschieden hübsch - eine gewisse zarte Erwärmung erfahren. Auch hierin war ich ,just another patient“. Wenn ich um eins oder zwei nicht mehr schlafen konnte und sie mir, mit einer Tasse Tee, die zweite Seconal-Kapsel brachte („die rote Kapsel“ hieß das vortreffliche, in Europa merkwürdigerweise unerhältliche Mittel natürlich nur), so erkundigte ich mich wohl nach ihrem Heim, ihrer Ausbildung, ihren Umständen. Sie war verlobt, oder verlobt gewesen, denn der Bräutigam hatte sich, wie sie achselzuckend berichtete, unsichtbar gemacht, war abgefallen. Warum denn nur? Ob sie etwa vermutete, daß er es mit einer anderen hielt? „Ich würde mich nicht wundern“, erwiderte sie. „Aber ich“, sagte ich, „ich würde mich höchlichst wundern über den Dummkopf! “ So weit ging ich, und sie lächelte herzlich. Sie verfügte auch über ein sehr lieb zuredendes Lächeln, wenn ich nachts, verdrießlich und störrig im Lehnstuhl kauernd, nicht mehr schlafen, nicht ins Bett zurückkehren wollte. Hatte sie mich dann begütigt und mir das stützende Kissen in den Rücken gestopft und den Lichtsignal-Druckkopf mit einer Sicherheitsnadel an meiner Decke befestigt, so beurlaubte sie sich für eine halbe Stunde zum Kaffeekränzchen der Nachtschwestern mit den Worten: „Now I am going to have my coffee“, wobei das „my coffee“ immer mit einer genießerischen Zärtlichkeit herauskam, an die zu denken mir noch heute Vergnügen macht.

So klassisch und jedes Zwischenfalls bar die Operation verlaufen war, so ereignislos, im klinischen Sinn, hurtig und ungestört ging es mit der Wiederherstellung voran. Ein Dreißigjähriger, versicherten die Ärzte, hätte sich nicht entgegenkommender verhalten können. Ich galt als eine Art „prize patient“. Der Chok, den 
jeder Eingriff dieser Art für den Gesamtorganismus, das Nervensystem bedeutet, war mir wohl fühlbar. Auch war eine Schwäche der Brust zurückgeblieben, die bei großer Neigung zu falschem Schlucken, das Räuspern und Aushusten ängstlich erschwerte. Obligate Verwachsungsschmerzen im Rücken wurden mit Kodein bekämpft, und die Veränderungen, die in meinem Innern, unter Entfernung der siebenten Rippe, vorgenommen worden, eine Höherlagerung des Zwerchfells und dergleichen, schufen nach vorschneller Bewegung wohl einige Atembedrängnis. Aber der Sauerstoff-Apparat, der eine Weile neben meinem Bett gestanden, verschwand sehr bald, und der meterlange Einschnitt heilte vortrefflich, so daß der hübsche Carlson (hübsche Menschen sind eine Freude, ob männlich oder weiblich) nach ein paar Wochen die Fäden entfernen konnte - mit einer Geschicklichkeit, die jede erwartete Unannehmlichkeit hintanhielt. Er war von der High School, deren Bildungsziele nichts Überspanntes haben, ohne Collegebesuch sogleich auf die Medical School gekommen, wo er übrigens als Marine-Aspirant seine Ausbildung gratis erhalten hatte, und wußte offenkundig in aller Welt von nichts etwas als von Chirurgie, für die er ebenso offenkundig geboren und in der er glücklich war. Noch sehe ich ihn in Gummihemd und Schürze eine Schubbahre auf Gummirädern mit einer lakenverhüllten Gestalt darauf in jungenhaftem Trab durch die Korridore von „Billings Hospital“ vor sich her treiben, - ein vergnügt einseitiges, gut anzuschauendes und tüchtiges Stück Leben.

Früh morgens, wenn June mich nach der Kunst im Liegen gewaschen und mir dann, bevor sie ging, eine Tasse Kaffee gebracht hatte (denn das Frühstück kam erst um 9 Uhr), setzte ich mich in meinem Schlafrock ans Fenster, betrachtete das Kommen und Gehen am großen Portal, sah nach den Fortschritten der sich begrünenden Bäume des Hofs und las unter Anstreichungen in Nietzsches Schriften, denn immer noch schwebte die schuldige „lecture“ über ihn mir als nächstes Agendum vor. Dann trat wohl Dr. Phemister, Vorsitzender der American Association of Surgeons und Chefarzt der Universitätsklinik, bester Typ amerikanischen Gelehrtentums, bei mir ein, erkundigte sich nach meiner Beschäftigung, blätterte in meiner Naumann'schen Nietzsche-Ausgabe und hinterließ einen oder den anderen medizingeschichtlichen Artikel, der seinen Verfassernamen trug. Adams und sein Gefolge kamen auf ihrem Rundgang zur Morgenvisite; es kam meine Frau, die Töchter kamen, und von auswärts fand mancher Besuch im Lauf des Tages, im Lauf der Tage sich ein: Bermann und Gumpert sprachen vor, Bruno Walter, der eben in Chicago konzertierte, saß an meinem Bett, Caroline Newton hatte gleichfalls die Fahrt von New York nicht gescheut und brachte Geschenke: ein Teegeschirr für den Nachmittag, eine Decke aus feiner Wolle. Alfred Knopf schickte Kaviar. Und an Blumen fehlte es nie. Drohten sie einmal auszugehen, so trat Erika mit frischen Rosen herein. In kritischer Lebenslage umgeben von so viel Liebe, Teilnahme, Fürsorge, fragt man sich, womit man sie verdient hat - und tut es ziemlich vergebens. War je einer, dem der Kobold des Hervorbringens im Nacken saß, so ein vom Jahr- und TagWerk immer Versorgter, Besessener, Präokkupierter - ein erfreulicher Mitmensch? Dubito. Und ich bezweifle es für mich persönlich noch ganz besonders. Wie ist es? Kann das Bewußtsein einer auf Konzentrationszerstreutheit beruhenden Unmenschlichkeit, kann etwa die Tönung der Existenz durch dieses Schuldbewußtsein selbst für mangelnde Leistungen aufkommen und versöhnend, ja Zuneigung gewinnend wirken? - Eine „Spekulation“, verrucht genug, um sie Adrian Leverkühn zuzuschreiben.
Der Roman - ich trug ihn in all diesen fremdartigen, abenteuerreichen Wochen fest im Herzen, stellte im Geist eine Liste notwendiger Verbesserungen an dem Geschriebenen her und dachte vorwärts. Mein Wohlverhalten als Patient, die meinem Alter kaum zustehende Behendigkeit im Genesen, die ich zeigte, dies ganze Bestehenwollen und glatte Bestehen einer späten und unerwarteten Belastungsprobe meiner Natur, - hatte es nicht alles ein heimliches Wozu?, stand es nicht in dessen Dienst, und brachte ich es nicht aus dem Unbewußten auf, um hinzugehen und dies fertig zu machen? Dabei war der Gedanke an das Werk wie eine offene Wunde, die nur, und sei es in liebevollster Absicht, berührt zu werden brauchte, um mich in meiner Schwäche auf unvorhersehbare Weise zu erschüttern. Meine Frau und Erika hatten in dem mitgeführten Maschinen-Manuskript gelesen, und während ich im Schlafrock appetitlos vor meinem schmalen Eßtischchen saß, sprach mir Erika von ihrem Eindruck durch einzelnes, nur von den ersten Besuchen der Freunde bei Adrian im Dorf, Spenglers, Jeanette Scheurls, Schwerdtfegers, von dem Kunstpfeifen Rudi‘s, und wie vorzüglich sie das alles finde. Sofort war ich in Tränen,- deren nichts als freudevollen Sinn ich dem Kinde klarzumachen hatte, da sie sich heftig wegen ihrer Unvorsichtigkeit schalt.

Vollständige Appetitlosigkeit war das einzige, worüber ich mich bei den immer gegenstandsloser werdenden Visiten der Ärzte noch zu beklagen hatte. Zum guten Teil war sie den ewigen, bis gegen das Ende des Aufenthalts fortgesetzten Penicillin-Gaben zuzuschreiben, diesem sicher preiswürdigen Schutzmittel, das aber auf die Dauer wie Harpyenunart die Speise schändet und, da die ganze Welt schließlich nach Penicillin riecht und schmeckt, größte Unlust zum Essen erzeugt. Eine gewisse kritische Heikligkeit der Sinne ist aber an und für sich diesem zarten Zustand eigen, der sich für vieles zu fein findet, was einem gröberen Dasein ansteht. Das zeigte sich in meiner mich selbst befremdenden Ablehnung geistiger Getränke. Der edelste Südwein, den Medi Borgese mir gleich ins Zimmer gestellt, war unannehmbar oder mundete doch nicht im geringsten. Selbst für das leichte amerikanische Bier fand ich mich zu gut. Was ich dagegen in Mengen, zu jeder Mahlzeit, trank, war Coca Cola, - dies populäre, aber freilich auch von Kindern bevorzugte Gebräu, an dem ich weder vorher noch nachher je Geschmack gefunden habe, das nun aber plötzlich mein ein und alles war.

Die Wiederkehr der Kräfte, ja der freien Bewegungsmöglichkeit wurde durch diese Launen und Verweigerungen des Organismus nicht aufgehalten. Wie schwer überwindlich schien erstmals die kurze Strecke von meiner Zimmertür rechtshin zu dem Gesellschaftsraum am Ende des Korridors! Bald legte ich am Arm meiner Frau oder der Nachmittagsschwester ein Vielfaches dieser Entfernung zurück auf den weit herumführenden Gängen des Stockwerks, wo immer aus den Lautsprechern die Namen irgendwo angeforderter Ärzte klangen. Es kam der Tag, an dem ich zum erstenmal wieder den Straßenanzug anlegte und, im Rollstuhl vor das Gebäude hinaus in die sich erwärmende Frühlingsluft befördert, auch etwas ausstieg, um eine mäßige Promenade in Front des Hauses zu halten, die Decke über den Knien auf einer Bank zu verweilen. Ich las viel während der langen Liegestunden. Anfangs war es die englische Ausgabe des gescheiten und vielgelobten Buches unseres Golo über Friedrich Gentz gewesen, die mich beschäftigte. Später liehen Borgeses mir die vier Bände des Grünen Heinrich, der mir sonderbarer-, ja skandalöserweise bis zur Stunde so gut wie unbekannt geblieben war. Ich kannte Kellers Korrespondenz mit dem Verleger Vieweg, der einen „Roman“ in Auftrag gegeben hat und nun fragt, drängt, ein solches Nicht- 
fertig-werden-können schlechthin nicht begreift, es für Trägheit hält und Betrug, schließlich in allem Ernst die Geduld verliert, während der junge Autor, dem etwas Einmaliges, aus aller Gewöhnlichkeit Fallendes, ein nur in Jahren auszuschöpfendes Werk eigenwilliger Größe unter den Händen wächst, sich entschuldigt, sich zu erklären sucht, keinen Termin halten kann und immer wieder um Zeit einkommen muß. Der hochkomische Konflikt hatte mir sehr gefallen, und doch hatte ich mich nie bemüßigt gefühlt, mit einem in so großer Tradition stehenden, meiner Sphäre so verwandten Werk eine mehr als oberflächlich versuchende Bekanntschaft zu machen. Hing es damit zusammen, daß meine Jugend sich viel mehr an „europäischer“, russischer, französischer, skandinavischer, englischer Literatur gebildet hatte als an deutscher, so daß ja auch die Begegnung mit Stifter sich erstaunlich lange verzögert hatte? Ich glaube, ich kannte von Kellers epischer Autobiographie nichts als eine oder die andere Jugend-Episode, nichts als Meierlein und seine „knappen Zifferchen“. Nun las ich mit wohligster Anteilnahme, mit immer wachsender Bewunderung für den reinlich ausgebreiteten Lebensreichtum des Buches, die köstliche Akkuratesse seiner aufs selbständigste an Goethe gebildeten Sprache, - mit Bewunderung, obgleich doch an der Ich-Figur der Erzählung, dem Grünen Heinrich eben, so wenig zu bewundern ist wie - gesetzmäßig offenbar - an den Helden anderer Erziehungs- oder Bildungsromane und der Name, den Goethe seinem Wilhelm einmal gibt: „Ein armer Hund“, noch besser auf ihn paßt, als auf diesen.

„You are still reading? You don't sleep? Shame on you!“ Es war June, die das sagte, wenn sie um 11 Uhr eintretend, noch Licht bei mir fand. Es wurde gelöscht, nur der bläuliche Schein des Nachtlämpchens blieb übrig, die seitliche Ruhelage war eingenommen und durchs Kissen befestigt, und der Nachtengel setzte sich zur Bewachung in den Stuhl, der am Tage auch mir nun so oft schon diente. Aber ich war dieses Daseins müde, durfte seiner müde sein, und in solcher Nacht entwarf ich den erregenden Plan, nicht volle sechs Wochen nach der Operation hier auszuharren, einen Übergangszustand zu schaffen und für die letzten Tage vor unserer Abreise ins Hotel, das vertraute Hotel Windermere nahe dem See, zu ziehen. Dr. Bloch wurde zitiert und befragt; er stimmte zu. Rasch waren die Vorbereitungen getroffen, es reihten sich herzliche Verabschiedungen, Bücher-Inskriptionen, Geschenke an die Pflegerinnen; und eine Presse-Konferenz war rasch noch angesetzt: eine Anzahl Journalisten hatte sich in einem unteren Gesellschaftsraum und Raucherzimmer versammelt, und an Erikas Arm, zum Reden noch keineswegs recht tüchtig, trat ich unter sie, - begierig eigentlich nur, das Lob der Anstalt, der Ärzte und der glorreichen Taten zu singen, die sie an mir verrichtet. Doch war gerade dies mir verwehrt, da „Billings Hospital“ keine „publicity“ duldet, auch mit Auskünften über mich in all der Zeit aufs äußerste sparsam gewesen war. So konnte ich den boys nur einiges Gutgemeinte über Politik vorsagen und wurde übrigens bald „cut short“ von Erika, die mit meinen Kräften sparte. Medi Borgese brachte uns in ihrem Wagen zum Hotel, wo sie Quartier gemacht hatte. Welche prächtigen Räume! Und die Mahlzeiten in unserer „dinette“, wieviel gewinnender als die Anstaltskost! Ich trank kein Coca Cola mehr. Dr. Bloch besuchte uns in der Freiheit. Der Eisenbahner-Streik verzögerte unsere Abreise um vierundzwanzig Stunden. Es gab viel Telephonieren der Feststellung wegen, ob und wann der „Chief“ nach Los Angeles fahren werde. Am Sonntag stand er bereit. Unter bequemsten Umständen, in einem „drawing-room“, mit privaten Mahlzeiten, vollzog sich die Rückreise. Dienstag den 28. Mai trafen wir zu dritt in Union Station wieder ein.

\section{Literatur}

1 Mann Th. Die Entstehung des Doktor Faustus. Roman eines Romans. Große kommentierte Frankfurter Ausgabe, Band 19,1: Essays VI Fischer Taschenbuch Verlag: Frankfurt am Main; 2012: 128 -144, Kapitel XII ab Seite 125, Zeile 30 und Kapitel XIII

2 Sprecher Th. Anmerkungen zu Thomas Mann und die Medizin. Praxis (Schweizerische Rundschau für Medizin) 2001; 90: 1235

3 Virchow Chr, Naef AP, Schaefer E et al. Thomas Mann (1875-1955) und die Pneumologie. Zur Indikation des thoraxchirurgischen Eingriffs im April 1946. Dtsch med Wschr 1997; 122: 1432 